\title{
NFC-Based Mobile Interactions with Direct-View Displays
}

\author{
Khoovirajsingh Seewoonauth ${ }^{1}$, Enrico Rukzio ${ }^{1}$, Robert Hardy ${ }^{1}$, and Paul Holleis ${ }^{2}$ \\ ${ }^{1}$ Computing Department, Lancaster University, UK \\ \{hardyr, rukzio\} @ comp. lancs.ac.uk \\ ${ }^{2}$ DoCoMo Euro-Labs, Germany \\ holleis@docomolab-euro.com
}

\begin{abstract}
Two NFC-based interactions are described in the paper. The first interaction technique is referred to as Touch \& Connect: a process by which an NFC tag is used to rapidly pair a mobile device with a computer. The second interaction technique is referred to as Touch \& Select, and considerably extends the Touch \& Connect concept by allowing the use of an NFC-enabled mobile phone to directly touch at, and select, an object on the computer screen. We achieve this by attaching a grid of NFC tags to the back of the screen. A picture browsing application has been developed in order to compare Touch \& Connect and Touch \& Select with the currently available Bluetooth-based approach. Our most salient findings show a considerable task time decrease for Touch-andConnect $(31 \%)$ and Touch-and-Select (43\%) over the standard Bluetooth approach for picture browsing tasks.
\end{abstract}

\section{Introduction and Overview}

The most prominent method of transferring data between a mobile device and computer is using Bluetooth. However, this involves performing various actions and can consume considerable time when pairing the devices for the first time. We provide a concept and a first implementation of two very quick and easy to use ways of sharing data between a mobile device and a computer system such as a laptop. Both interactions use passive NFC (Near-Field Communication) tags that allow a small amount of data to be read / written to them. In order to test the new types of interaction, with respect to each other and to other possibilities, a picture sharing application was developed. The application allows the downloading and uploading of pictures between the laptop and the mobile device. In addition, the laptop display can be used to enlarge and view pictures in full resolution.

\section{Related Work}

Existing work has mainly focused on augmenting static user interfaces such as maps [1] or posters [2] with NFC tags. The disadvantage of this approach is that only the mobile phone can provide feedback. This approach was then extended through the use of NFC with front-projection displays. As shown in [3], a grid of tags can then be 
used to connect virtual content to the tags and display dynamic visual feedback. However, besides difficulties with the image quality of projections in bright light conditions, a big disadvantage of such a setup is the occlusion of the image, which is caused by people and objects (such as the phone used to touch the tags). By extending NFC deployment to direct-view displays (as presented in this paper), visual feedback can be maintained in almost all light conditions. Consequently, the problem with occlusion is also nearly negligible. The tag mesh is attached to the screen itself, thus improving portability and requiring considerably less physical space. Moreover, in contrast to projection, there are also no deployment issues with regards to calibration.

\section{Implementation and Use}

The presented system is the first to implement an interaction technique in which a mobile phone can directly interact with a direct-view display through intuitive touch interaction without the problems that would exist when using a front-projection display. We used a MacBook laptop and a Nokia 6131 NFC phone for the implementation of the prototypes.

\subsection{Touch and Connect}

Touch \& Connect is a technique in which a Bluetooth connection between a mobile phone and laptop is established by simply touching the laptop with the mobile device. The NFC tag, used for initiating the connection, can additionally be employed for transferring extra data between the devices.

For this prototype, an NFC tag is attached to the laptop's armrest and stores the laptop's Bluetooth MAC address which is then used to avoid the time required for searching available Bluetooth devices. Subsequent connections to the NFC tag are then used to initiate actual data transfers between the devices. This also eliminates problems concerning searching for previously paired devices as the device names can be ambiguous. Using the NFC tag when transferring data, the user can directly specify the target device which can be automatically inferred by the application.
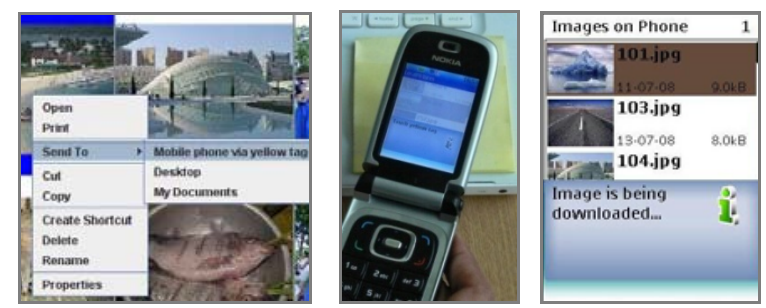

Fig. 1. Downloading a picture from the laptop to the mobile phone with Touch \& Connect: select the appropriate option for a picture and touch the tag on the laptop with the phone

To upload a picture, the user first selects a picture on the phone, then chooses upload. The mobile phone informs the user to touch the tag on the laptop in order to upload the image. The picture is then promptly copied and displayed on the laptop. 
Reversely, in order to download an image to the phone, it is selected using the mouse in the picture browsing application on the laptop. Then, the user chooses "Send To Mobile Phone via Yellow Tag" (Fig. 1, left). The user then touches the yellow tag on the laptop (Fig. 1, center) to copy the image to the phone (Fig. 1, right).

\subsection{Touch and Select}

The concept of Touch \& Connect is extended by transferring information and data via multiple tags. The major advantage is that the user can specify the screen location where information is to be sent or read. It can also simultaneously provide multiple options to the user. As shown in Fig. 2 (left), the Touch \& Select prototype features a laptop display; the back of which was augmented with a mesh of 7x4 NFC tags. Each tag stores its location in the mesh together with the Bluetooth address that resolves to the laptop Bluetooth device. The location coordinates are shown in Fig. 2 (center). The mobile phone can read this information when touching the front of the laptop display (see Fig. 2, right). Through this the user can select any object (e.g. a picture or folder) shown on the laptop display by touching it with the mobile phone.

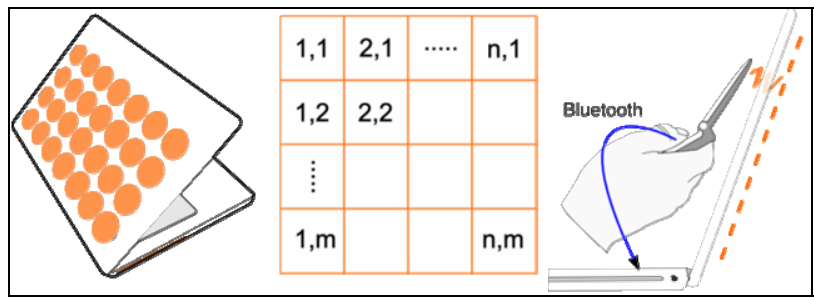

Fig. 2. Touch and Select. Left: NFC tag grid attached to the back of a laptop screen. Center: location information stored in the tags. Right: interaction with the NFC tags on the laptop.
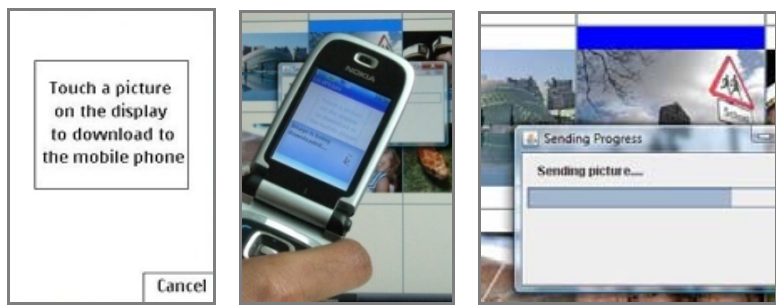

Fig. 3. Downloading a picture from the laptop to the mobile phone with Touch \& Select

The picture browsing application has been implemented in such a way that each photo item corresponds in its position to a tag in the back of the display. Even though this mapping suggests itself for this application, we argue that this is also possible for other types of applications based on sharing of information. With smaller tags coming on the market, the grid granularity can be increased such that a more precise location of the phone can be tracked. To upload a picture with Touch \& Select, the user first selects it on the phone and chooses upload. The mobile phone then informs the user 
that an empty square on the display can be touched in order to upload the image. When a user touches the display, the corresponding picture is uploaded and shown on the screen of the laptop. The advantage of this method, besides being able to quickly transfer the file, is that a concrete location (or potentially application) can directly be chosen on the screen. Similarly, in order to download an image, the user switches to download mode on the mobile phone (Fig. 9, left) and selects the picture on the laptop by touching it with the mobile phone (Fig. 9, center). The picture is then copied to the mobile phone (Fig. 9, right) and displayed there. Again, the advantage is that the image can directly be selected using the phone and no additional interaction is necessary saving extra steps and considerably increasing the speed for this interaction.

\subsection{Comparative Study and Outlook}

In order to compare the new techniques with existing methods, we also implemented picture transfer via the standard Bluetooth method using the same picture sharing application interface as described above. The user selects a picture on the phone and selects "Send via Bluetooth". The phone then searches for available Bluetooth devices and presents a corresponding list. After choosing the entry for the laptop, the image is copied and displayed in the picture browsing application running on the laptop. Downloading is implemented accordingly.

In a study (with 19 participants) we compared Touch \& Connect and Touch \& Select with conventional Bluetooth-based file transfers [4]. This study showed that Touch \& Select is significantly better than Touch \& Connect, which is again significantly better than the currently used Bluetooth-based approach when considering user preferences, task completion time, usability satisfaction, task load and qualitative feedback from the study participants. Specifically, task completion time for uploading and downloading pictures was $31 \%$ (using Touch \& Connect) and $43 \%$ (using Touch $\&$ Select) faster than the conventional Bluetooth-based approach.

The presented interaction techniques - Touch \& Connect and Touch \& Select significantly simplify the way data can be transferred between a mobile phone and a computer.

Embedding NFC tags into TFT / LCD / Plasma / rear-projection displays has the possibility to provide many opportunities for developing novel and exciting interaction techniques enhancing the human computer interface by using the mobile phone as a smart input device. Further application areas that could be investigated include mobile device synchronization, general file transfer, and uploading contextual information from the phone. With regards to further deployment options, the tag mesh could be augmented to digital picture frames and public displays.

\section{References}

1. Reilly, D., Rodgers, M., Argue, R., Nunes, M., Inkpen, K.: Marked-up Maps: Combining Paper Maps and Electronic Information Resources. PUC 10(4), 215-226 (2006)

2. Rukzio, E., Broll, G., Leichtenstern, K., Schmidt, A.: An Evaluation and Comparison of Physical Mobile Interaction Techniques. In: AmI 2007 (2007)

3. Hardy, R., Rukzio, E.: Touch \& Interact: Touch-based Interaction with a Tourist Application. In: MobileHCI 2008, pp. 531-534 (2008)

4. Seewoonauth, K., Rukzio, E., Hardy, R., Holleis, P.: Touch \& Connect and Touch \& Select: Interacting with a Computer by Touching it with a Mobile Phone. In: MobileHCI 2009 (2009) 